\title{
Targeted delivery of doxorubicin to HER2 positive tumor models
}

Hosna Gomari'

Mehdi Forouzandeh Moghadam'

Masoud Soleimani ${ }^{2}$

Mahlegha Ghavami'

Shabanali Khodashenas ${ }^{3}$

'Department of Medical Biotechnology, Faculty of Medical Sciences, Tarbiat Modares University, Tehran, Iran; ${ }^{2}$ Department of Hematology, Faculty of Medical Sciences, Tarbiat Modares University, Tehran, Iran; ${ }^{3}$ Molecular and Cell Biology Research Center, Faculty of Medicine, Mazandaran University of Medical Sciences, Sari, Iran

Correspondence: Mehdi Forouzandeh Moghadam

Department of Medical Biotechnology, Faculty of Medical Sciences, Tarbiat

Modares University, Jalal ale Ahmad

Highway, Tehran, IR, Iran

Tel +982182883861

Fax +98218288386

Email foroz@modares.ac.ir
This article was published in the following Dove Press journal:

International Journal of Nanomedicine

Background: Exosomes are natural nanovesicles with unique characteristics, such as long circulating half-life, the intrinsic ability to target tissues, biocompatibility, and minimal or no inherent systemic toxicity. Mesenchymal stem cells produce large amounts of exosomes with regenerative properties and more stability in human plasma. TUBO breast cancer cell lines overexpress rat HER2/neu protein.

Methods: Targeted exosomes were isolated from transduced bone marrow mesenchymal stem cells. Doxorubicin was encapsulated into exosomes by electroporation. Flow cytometry was used to assess the attachment of exosomes to the target cells. The in vitro cytotoxicity effect of targeted doxorubicin-loaded exosomes on TUBO cells was determined using MTT assay. Selective delivery of doxorubicin to tumor tissues was analyzed by measuring the auto-fluorescence of doxorubicin by in vivo imaging system. Moreover, tumor growth inhibition and body weight were monitored following injection of free doxorubicin, and targeted and untargeted doxorubicin-loaded exosomes in a TUBO breast cancer model. Finally, mouse tissues were examined for the presence of intrinsic fluorescence of doxorubicin.

Results: Flow cytometry results revealed significant differences in binding of targeted exosomes to HER2-positive (46.05\%) and HER2-negative (13.9\%) cells. The results of MTT assay showed that cytotoxicity of targeted doxorubicin-loaded exosomes was higher than free doxorubicin at 72 hours. Selective distribution of targeted doxorubicin-loaded exosomes in the target tissues of the murine breast cancer model suggested specific delivery of doxorubicin by targeted exosomes, rather than untargeted exosomes. Free doxorubicin and untargeted doxorubicin-loaded exosomes showed insignificant effects, whereas targeted doxorubicin-loaded exosomes reduced the tumor growth rate.

Conclusion: Herein, we report efficient delivery of targeted doxorubicin-loaded exosomes in vitro, corroborated with a significant reduction of murine breast cancer model tumor growth rate.

Keywords: exosome, breast cancer, TUBO, targeted therapy, drug delivery

\section{Introduction}

Cell communications occur in two ways: distant communication or localized. Distant intercellular communication can take place by either hormones or extracellular vesicles (EV) through the circulatory system effecting the other parts of the body. Extracellular vesicles have a bilayer membrane structure and serve as vehicles to deliver different kinds of cellular cargo, including proteins, lipids, nucleic acids, and receptors (1). Therefore, EVs can transfer information between tissue microenvironments.

Exosomes are originated from endosomes with a small size ranging from 40-100 nm (1). Depending on their origin, these nanoparticles may contain endosomal membrane, 
fusion (GTPases, flotillin, and annexins) and tetraspanin proteins (CD81, CD63, CD53, CD82, and CD37). Other proteins present in exosomes are the ones associated with lipid rafts, including glycosyl phosphatidylinositol-anchored proteins, heat shock, and proteins related to multi-vesicular body (MVB) biogenesis (eg, TSG101 and Alix) (2).

Exosomes embed and shield a large number of proteins, lipids, mRNAs, and miRNAs, which allow them to influence the function and differentiation of recipient cells (3). They show biocompatibility characteristics such as immune tolerance, enabling them to escape from the immune system. Previous studies demonstrated that near neutral, tiny negative zeta potential charge of exosomes is responsible for higher in vivo circulation and stability of these nanoparticles compared to positively charged liposomes. ${ }^{1,2}$ Positive zeta potential leads to the aggregation of liposomes with negative particles in blood circulation and reduces their flowing time and, therefore, decreases their accession to target sites.

For more efficient targeting, exosomes can be modified to serve as nano delivery systems either endogenously at the production time or exogenously following the exosome isolation. ${ }^{1}$ Doxorubicin (DOX) is one of the most effective antitumor drugs against solid tumors including breast cancer, but the clinical usage has been limited due to its low bioavailability and severe side-effects, such as myelo suppression and cardiotoxicity. Loading of doxorubicin in nanoparticles increases the rate of delivery and antitumor activity. ${ }^{3-5}$ However, synthetic nanoparticles have some adverse effects, including the induction of immune responses and oxidative stress. ${ }^{6}$ As natural nanoparticles with small size, exosomes are good candidates for drug delivery. Successful delivery of therapeutic cargo by exosomes is highly dependent on the efficiency of the loading method. In order to avoid the adverse side-effects of chemotherapeutic drugs, it is imperative to deliver them specifically to the target tissue for cancer treatment. Using targeting peptides or proteins on the surface of exosomes is the most common approach for selective delivery. ${ }^{7}$

In clinical applications, the source of exosomes is important. Previous studies recommend mesenchymal stem cells (MSCs) as an efficient source of exosomes, due to their stability, considerable exosome production potential, and relatively high-tolerance. ${ }^{8}$ Moreover, the homing ability of exosomes allows them to migrate towards injured tissue and tumor, which is due to the acidic $\mathrm{pH}$ of the tumor microenvironment. Chemokines such as CCL2, CCR8, platelet-derived growth factor
(PDGF), and tumor necrosis factor-a (TNF-a) have recently been recognized as chemotaxis particles with important roles in the attraction of MSC mediators to tumor environment. ${ }^{9}$

Previously, we designed targeted exosomes with a chimeric protein against HER2-positive breast cancer. ${ }^{10}$ These targeted exosomes were used for the delivery of siRNA to breast cancer cells. ${ }^{11}$ The targeted exosomes were also employed to deliver doxorubicin to HER2-positive cancer cells. ${ }^{12}$ In the present study, we aimed to evaluate anti-cancer effects of targeted exo-DOX in a murine tumor model inoculated with TUBO cells.

\section{Materials and methods}

\section{Cell culture}

The TUBO cell line was kindly provided by Dr. Pier-Luigi Lollini (Department of Clinical and Biological Sciences, University of Turin, Orbassano, Italy) and cultured in DMEM medium supplemented with $20 \%$ FBS and pensilin/streptomycin antibiotics, and incubated at $5 \% \mathrm{CO}_{2}$ at $37^{\circ} \mathrm{C}^{13,14}$

\section{Viral production and transduction of LAMP2b-DARPin}

HEK293T cell lines were seeded $\left(4 \times 10^{6}\right.$ cells/plate) on $10-\mathrm{cm}$ diameter plates. After 24 hours, calcium phosphate transfection was performed by adding $10.5 \mu \mathrm{g}$ of pMD2 (addgene), $21 \mu \mathrm{g}$ of psPAX2 (addgene), and $21 \mu \mathrm{g}$ of pLEX-LAMP2bDARPin.

The condition media containing viral particles was harvested every 24 hours up to 72 hours. Supernatant was centrifuged at $500 \mathrm{~g}$ for 5 minutes and the cell pellet was discarded. The harvested supernatant was subsequently centrifuged $(2,000 \mathrm{~g}$ for 20 minutes) and then filtered through a $0.45-\mu \mathrm{m}$ PVDF (low protein attachment) membrane. To concentrate viral particles, centrifugation at 19,000 $\mathrm{g}$ for 1:30 hours was applied and MOI (number of viral particles per target cell) was measured for MSCs transduction.

After production of virus particles containing the LAMP2b-DARPin construct, MSCs were transduced by viral particles as previously described. ${ }^{12}$ Briefly, $2 \times 10^{4}$ MSCs per well were seeded in a 24 -well plate. Viruses with MOI of $20-100$ and $100 \mu \mathrm{g} / \mathrm{ml}$ protamine sulfate were added to each well. This procedure was repeated three times to increase viral transduction efficiency. After 1 week, the selection process was performed using an 
appropriate concentration of puromycin $(1 \mu \mathrm{g} / \mathrm{ml})$ and transduced MSCs were passaged to obtain exosomes. Subsequently, RT-PCR and Western blotting were performed to confirm the transcription and translation of LAMP2b-DARPin (data not shown).

\section{Purification of exosomes}

In order to isolate untargeted and targeted exosomes, MSCs and transduced MSCs were cultured in serum-free media for 48 hours after reaching $80 \%$ confluency, and the supernatant was harvested to extract exosomes by cell guidance biosystem exosome purification kit, as previously described. ${ }^{12}$ In brief, the supernatant was centrifuged to eliminate cell debris. After filtration through $0.22 \mu \mathrm{m}$ filters, the media was concentrated using 100-kDa MWCO columns (Merck Millipore, UK). The rest was carried out according to the manufacturer's instructions.

\section{Exosome labeling and uptake study}

Exosomes were labeled with PKH67 (Sigma-Aldrich, St. Louis, MO, USA), which is a green lipophilic dye. Exosomes and PKH67 were separately diluted in $100 \mu \mathrm{L}$ diluent $\mathrm{C}$. Exosomes were mixed with staining solution and incubated for 5 minutes at room temperature. Stopping process was done by adding an equal volume of $1 \%$ BSA and the mixture was passed through a CL-4B shepharose column to remove unincorporated dye. TUBO cells were seeded in a 24 -well plate $\left(2 \times 10^{4}\right.$ cells/well), and $25 \mu \mathrm{g}$ of labeled exosome was added to each well. After 24 hours, the cells were washed with PBS and fixed by 4\% paraformaldehyde. The nuclei were stained by DAPI fluorescent (Sigma-Aldrich), and examined using an inverted fluorescence microscope (Nikon TE300, Tokyo, Japan).

\section{Flow cytometry and fluorimetry}

We used flow cytometry to quantify exosome uptake. Briefly, 4-6×10 4 TUBO and 4T1 cells were seeded in 24-well plates, and $5 \mu \mathrm{g}$ of labeled exosomes were added to each well. After 24 hours, cells were washed with PBS and trypsinized. ${ }^{12,15}$ Flow cytometry was performed by BD flow cytometry and data were analyzed by flowing software 2.4.1. 4T1 cells were used as control cells.

Binding of PKH-67 labeled targeted and untargeted exosomes to the HER2 protein coated wells were assayed by measuring the fluorescence of each well in black wells, after removing unbound exosomes. BSA and PBS were used as controls. Briefly, 80-90 ng of HER2 protein was added in each 96 well plate, after overnight incubation at $37^{\circ} \mathrm{C}$, labeled exosomes were added with different concentration $(0.05,0.1$, and $0.2 \mu \mathrm{g} / \mu \mathrm{L})$. Following overnight incubation, the attachment of labeled exosomes with the HER2 protein was determined. Attachment of labeled exosomes with the HER2 protein was determined at different concentrations $(0.05,0.1$, and $0.2 \mu \mathrm{g} / \mu \mathrm{L})$. Next, HER2-positive SKBR3 cells were seeded and fixed in a 96-well black plate and 0.05, 0.1, and $0.2 \mu \mathrm{g} / \mu \mathrm{L}$ of targeted and untargeted exosomes were added to these wells in triplicate and incubated overnight. Fluorescence emission of PKH-67 labeled exosomes was measured after removing non-labeled exosomes by washing with PBS and calculated by Microplate Readers (BioTek, Winooski, VT, USA) at $520 \mathrm{~nm}$ (excitation at $480 \mathrm{~nm}$ ).

\section{Loading therapeutic cargo}

To load exosomes with doxorubicin (Sigma-Aldrich), $100 \mu \mathrm{g}$ (total protein) of purified exosomes and $100 \mu \mathrm{g}$ of DOX were gently mixed with $200 \mu \mathrm{L}$ of electroporation buffer at $4^{\circ} \mathrm{C}$. Electroporation was performed at $600 \mathrm{~V}$ in $0.4 \mathrm{~cm}$ cuvettes in a multiporator (Eppendorf, Hauppauge, NY, USA) and the mixture was incubated at $37^{\circ} \mathrm{C}$ for 30 minutes to recover the membrane of exosomes. ${ }^{16}$ After passing through a 100-KDa Amicon filter to eliminate free DOX, drug loading was measured through the intrinsic fluorescence of DOX using a spectrophotometer (Bio-Rad, Hercules, CA, USA) at $594 \mathrm{~nm}$ with excitation at $480 \mathrm{~nm}$.

\section{In vitro cytotoxicity}

Cytotoxic activity of free DOX and exo-DOX was evaluated by standard 3-(4,5-dimethyl-2-thiazolyl)-2,5-diphenyl-2-H-tetrazoliumbromide (MTT) assay on TUBO cells. The MDAMB231 cell line was used as control (data was not shown). ${ }^{12}$ TUBO cells $(5,000$ cells/well) were added to $100 \mu \mathrm{L}$ of media in a 96-well plate overnight. Then, the cells were treated with various concentrations of exo-DOX for 24,48 , and 72 hours at $37^{\circ} \mathrm{C}$ and $5 \% \mathrm{CO}_{2}$. After incubation, media containing drug were replaced and cells were incubated with MTT reagent for 34 hours. Subsequently, MTT reagents were removed completely and $100 \mu \mathrm{L}$ of DMSO was added to each well to dissolve the solubilized purple formazan crystals. ${ }^{17}$ Absorbance of the colored solution was quantified by a spectrophotometer at 545/ $630 \mathrm{~nm}$. Cytotoxicity rates were measured in comparison to negative control (wells containing only PBS). All experiments were performed in triplicate and IC50 of loaded exosomes and free DOX were assessed and compared to each other. ${ }^{18}$

\section{Immunohistochemistry}

Murine HER2-positive TUBO cells were injected subcutaneously to $\mathrm{BALB} / \mathrm{c}$ mice. After tumor genesis, the tumor 
was fixed in $10 \%$ formalin and immunohistochemistry (IHC) was done to confirm the presence of cell surface HER2 ligands.

\section{Mouse xenograft and in vivo studies}

Female B6 nude mice (5 weeks old) were purchased from Royan Institute. Mice received a sterilized diet and filtered water under standard conditions according to institutional guidelines. All procedures were approved by the Institute's Ethical Committee for animal use.

Each mouse was inoculated by subcutaneous injections in the flank with $1 \times 10^{6}$ TUBO cells suspended in $0.1 \mathrm{~mL}$ of PBS and were allowed to develop a $100-\mathrm{mm}^{3}$ tumor. The mice were randomly assigned to four groups of four tumors for each group and were treated with: (a) PBS as a control, ${ }^{19}$ (b) untargeted exo-DOX (30 $\mu \mathrm{g}$ DOX/mouse), (c) targeted exo-DOX (30 $\mu \mathrm{g}$ DOX/mouse), and (d) free DOX $(30 \mu \mathrm{g} /$ mouse). All treatments were administered intravenously and repeated twice a week and continued for six injections. To monitor the effect of treatment on each group, the tumor size and mice weight were measured twice a week after each injection. Tumor volumes were measured by digital caliper with the following formula: ${ }^{20,21}$

$$
\text { Volume }=\left(\text { length } \times \text { width }^{2}\right) / 2
$$

\section{In vivo exosomes distribution}

When the TUBO tumor volume reached $>400 \mathrm{~mm}^{3}$, targeted and untargeted exo-DOX (70 $\mu \mathrm{g} /$ mouse) were injected intravenously to a B6 nude mice breast cancer model. PBS was injected in the control group. Mice bearing xenograft tumor were anesthetized,and images were acquired 30 minutes and 60 minutes post-injection by Kodak in vivo imaging system $\mathrm{F}$ pro. Following the last acquisition, the animals were sacrificed and the organs (liver, spleen, heart, and tumor) were collected, washed, and captured using the same imaging system. ${ }^{20}$

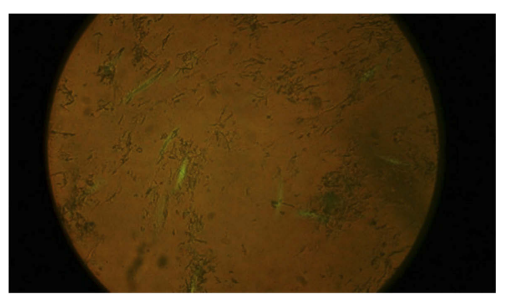

A

\section{Statistical analysis}

Assays were performed in triplicate, and data are presented as the mean $( \pm$ standard deviation, SD) of three independent experiments. Data were analyzed using GraphPad Prism 6.07 software (GraphPad Software Inc.). Results were evaluated using two-way ANOVA and differences between groups were calculated by the Student's $t$-test. Two asterisks $\left({ }^{* *}\right)$ stand for $P<0.005$ and one asterisk $\left(^{*}\right)$ denotes $P<0.05 .{ }^{19}$

\section{Results}

\section{MSCs isolation and transduction}

Viral particles containing the LAMP2b-DARPin gene and the others with GFP gene were added simultaneously to different cell cultures, and transduced cells were selected by an adequate concentration of puromycin $(1 \mu \mathrm{g} / \mathrm{mL})$. GFP expression was determined by fluorescent microscopy (Figure 1). Due to the absence of a GFP reporter gene, integration of LAMP2bDARPin was analyzed by RT-PCR, and viral titration was calculated by quantitative real-time PCR.

Furthermore, a 70-kD protein (LAMP2b-DARPin) was identified by Western blotting using an anti-His tag antibody. ${ }^{12}$

\section{Exosome characterization}

The concentration of exosomes were calculated by Bradford assay, and approximately $200 \mu \mathrm{g}$ of exosome was obtained from $120 \mathrm{~mL}$ of conditioned media. TEM analysis verified the cup-shaped appearance of isolated MSCs-Exo with an average size of 30-150 nm (Figure 2A). Expression of CD9, CD81 and CD63 exosomal markers, and the absence of calnexin (an endoplasmic reticulum marker), were observed using enhanced chemiluminescence (ECL) Western blot analysis (Figure 2B). The results obtained from zeta sizer exhibited $120 \mathrm{~nm}$ as the average size of MSCs-derived exosomes (Figure 2C).

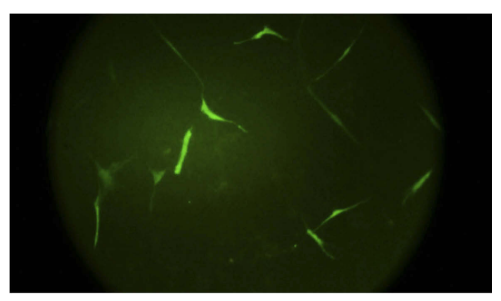

B

Figure I MSCs transduction with viral GFP. (A) Fluorescent and phase contrast images were merged. (B) Fluorescent image of the same frame. Low efficiency of transduction can be observed.

Abbreviations: MSCs, mesenchymal stem cells; GFP, green fluorescent protein. 


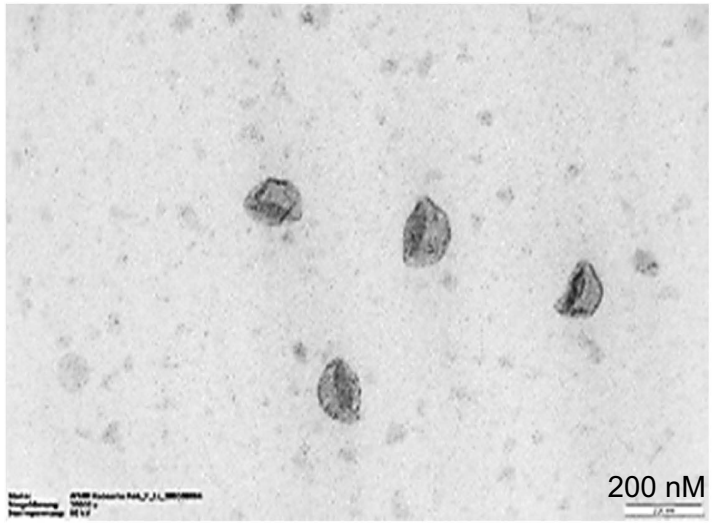

A

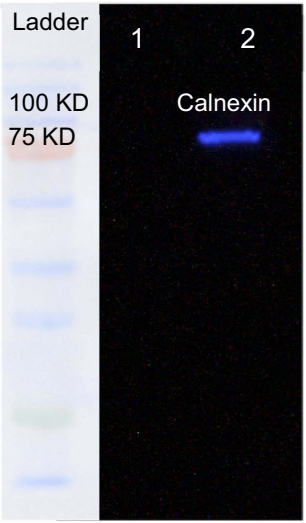

Size distribution by intensity
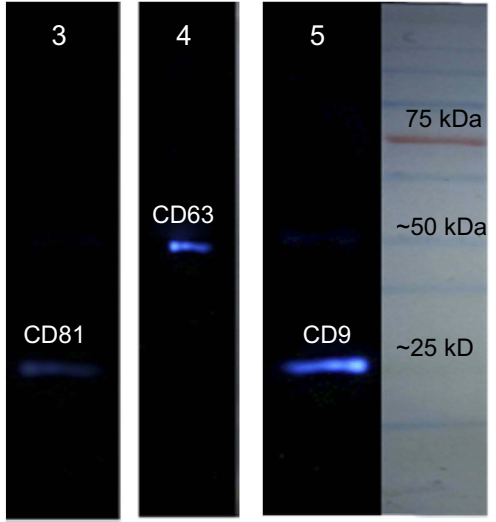

B

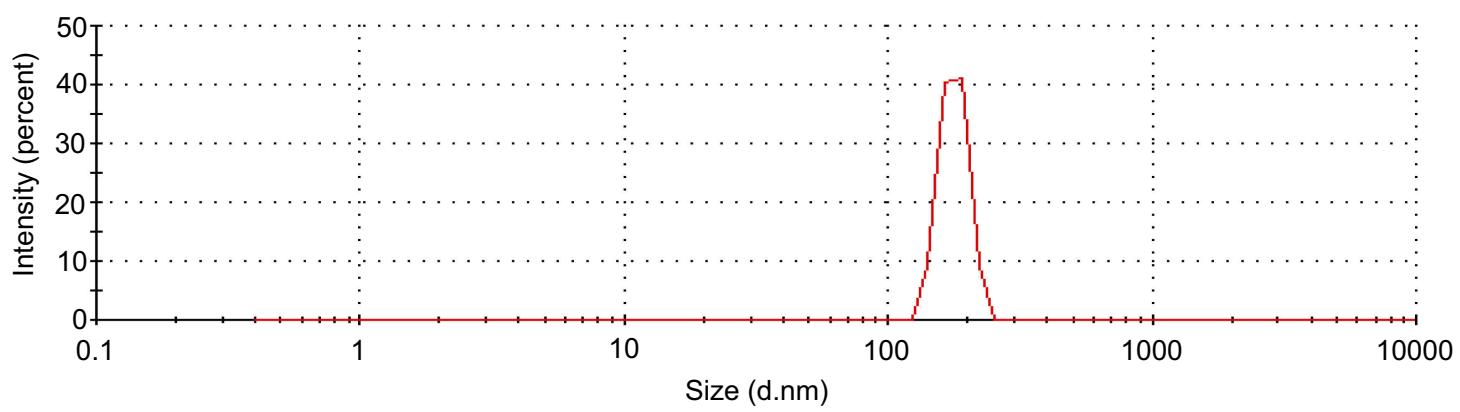

C

Figure 2 Characterization of exosomes. (A) TEM images of exosomes. Typical structure of exosomes was shown in this image (scale bar $200 \mathrm{~nm}$ ). (B) Characterization of exosomes by ECL Western blotting. Exosome preparations were negative for endoplasmic reticulum marker (calnexin) in lane I; lane 2 is the cell lysate which is positive for calnexin. The positive results for exosomal markers are displayed in lane 3 (CD8I), lane 4 (CD63), and lane 5 (CD9). (C) Size distribution of exosomes was measured by a zeta-sizer. The peak diameter was about $100 \mathrm{~nm}$.

Abbreviations: ECL, enhanced chemiluminescence; TEM, transmission electron microscopy.

\section{Flow cytometry and fluorimetry}

To discriminate whether LAMP2b-DARPin-targeted exosomes can bind more efficiently to HER2 protein, fluorimetric analysis was performed. Attachment of PKH-67 labeled exosomes to immobilized HER2 protein was determined at different concentrations of targeted and untargeted exosomes. Results showed a significant difference in binding of targeted exosomes compared to untargeted exosomes at all tested concentrations. PBS and BSA were used as negative control (Figure 3A). These results were established by comparing specific binding of targeted and untargeted exosomes to HER2-positive SKBR3 cells. However, significant discrepancy was only observed at $0.05 \mu \mathrm{g} / \mu \mathrm{L}$ and $0.1 \mu \mathrm{g} / \mu \mathrm{L}$ concentrations (Figure 3B).

TUBO cancer cells (murine HER2-positive cell line) and 4T1 (murine HER2-negative cell line) were treated with targeted and untargeted exosomes previously labeled with PKH67. As demonstrated in Figure 3C, targeted exosomes bound to TUBO cells (46.6\%) more efficiently compared to 4T1 cells (13.9\%). Untargeted exosomes were used as exosome binding control. ${ }^{12}$ A fluorescent image of targeted exosomes labeled with PKH-67 and TUBO cells is illustrated in Figure 4.

\section{Cell treatment and cytotoxicity of loaded DOX}

Electroporation was used to load DOX into exosomes. The amount of encapsulated DOX was determined by measuring the auto-fluorescent property of DOX at $595 \mathrm{~nm}$ (excitation at $480 \mathrm{~nm}$ ) against a serial dilution of known standards. Our results showed that $\sim 13 \%$ of DOX were loaded into the exosomes. TUBO cells were treated with exo-DOX. Figure 5 shows that exo-DOX can enter the cells and accumulate in nuclei (Figure 5A). Next, TUBO cells were treated with different concentrations of exo-DOX and free DOX, and the cytotoxic effect of free DOX and DOX-loaded exosomes was examined. Free DOX and exo-DOX considerably reduced cell viability in a dose-dependent manner, and no significant difference was observed at 24 hours and 48 hours, while significant variances were recorded at 72 hours (Figure 5B). 


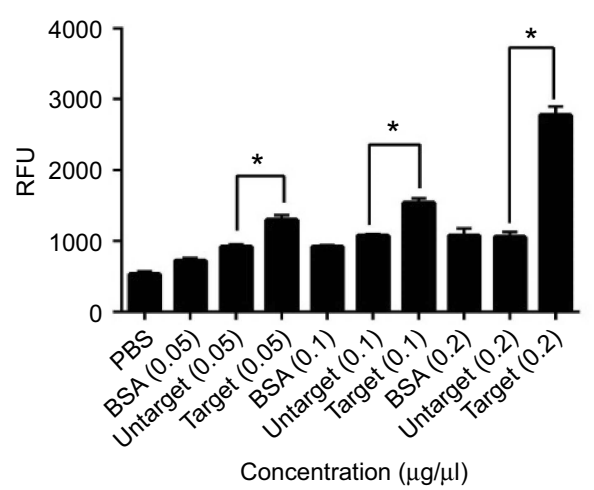

A
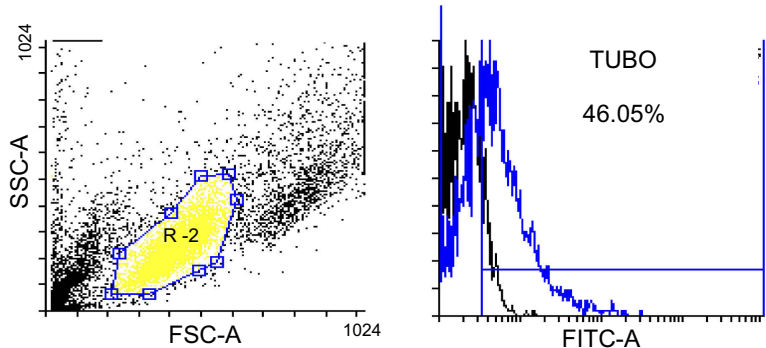

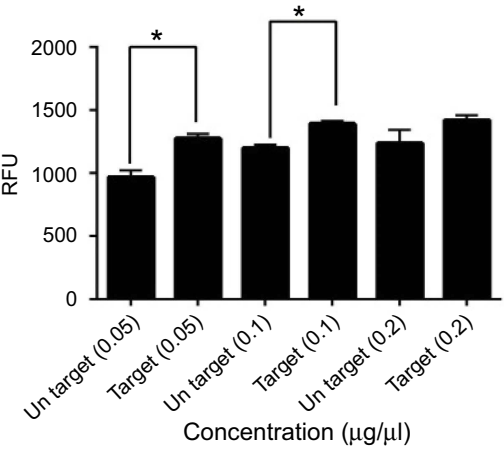

B
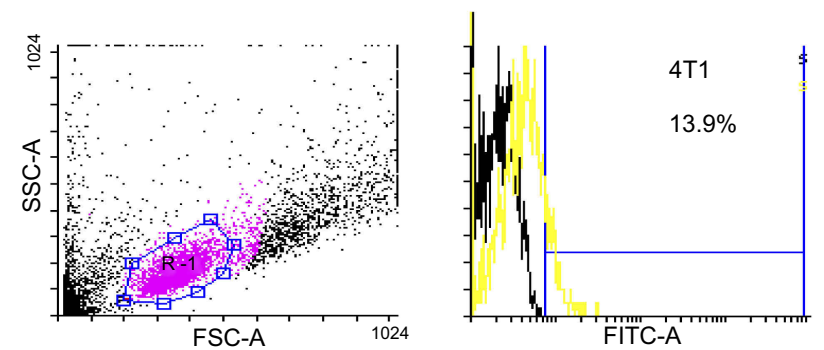

C

Figure 3 Fluorimetry and flow cytometry analysis of binding ability of targeted and untargeted exosomes. Binding of PKH-67 labeled targeted and untargeted exosomes to (A) coated HER2 protein and (B) HER2-positive SKBR3 cells with different concentrations. Fluorescence of each well was assessed by fluorimetry after removing unbound exosomes. BSA and PBS were used as controls. (B) Binding of PKH-67 labeled targeted and untargeted exosomes to HER2-positive SKBR3 cells. There were significant differences both in HER2 protein and HER2-positive cell immobilization; yet, no significant difference was observed at $0.2 \mu \mathrm{g} / \mu \mathrm{L}$ in binding to SKBR3 cells. (C) Binding and entrance of targeted labeled exosomes were quantified by flow cytometry. The results show a significant increase in binding of targeted exosomes to TUBO cells (46.05\%) as HER2-positive murine cells compared to 4TI cells (I3.9\%). Targeted exosomes showed significantly higher binding to HER2-positive cells compared to HER2-negative cells. Each error bar represents the mean $\pm S D$ of three replicates. $* P<0.05$.

Abbreviations: RFU, relative fluorescence units; BSA, bovine serum albumin.

\section{Animal studies}

Immunohistochemistry of the TUBO cell line

The tumor produced by injection of BALB/c mouse with TUBO cells was fixed in $10 \%$ formalin and IHC was performed, which diagnosed the tumor as HER2++ (Figure 6).

\section{In vivo distribution of exosomes}

Tumor entrancement and biodistribution of exo-DOX in B6 nude mice bearing TUBO tumor were observed by in vivo imaging system (IVIS). Intrinsic fluorescent intensity of DOX in injected mice was determined 30 minutes and 60 minutes post-injection. PBS was injected as control. As shown in Figure 7, the fluorescent intensity of exo-DOX can be observed at different tissues of mice as well as tumor sites, in tumor bearing mice. Fluorescent intensity significantly increased in the tumor site at 60 minutes (Figure 7A) in comparison to 30 minutes (Figure 7B), in both targeted and untargeted exosomes; however, the intensity of untargeted exosomes was obviously lower than targeted exosomes at the tumor site.
All mice, including control mice, were sacrificed after 60 minutes and a fluorescent signal was immediately detected from freshly dissected tissues using an in vivo imaging system. Fluorescent intensity of exo-DOX was readily observed in the tumor tissue along with most tissues of the body, including the lung and liver (Figure 7C).

\section{The effect of exo-DOX in B6 nude mice bearing TUBO tumor}

In this study, we had four groups, and each group included four tumors. The first group were injected by targeted exoDOX $(1.5 \mathrm{mg} / \mathrm{kg})$, whereas the second group was administered with untargeted exo-DOX $(1.5 \mathrm{mg} / \mathrm{kg})$; the third group was injected with an equivalent amount of free Dox, and the fourth with PBS as negative control. After each injection, the mice were weighed and tumor volume was measured. Tumor growth was assessed twice per week and continued for five injections. Although tumors continued to grow, the tumor of mice receiving targeted exo-DOX grew more slowly compared to those receiving exo-DOX, 


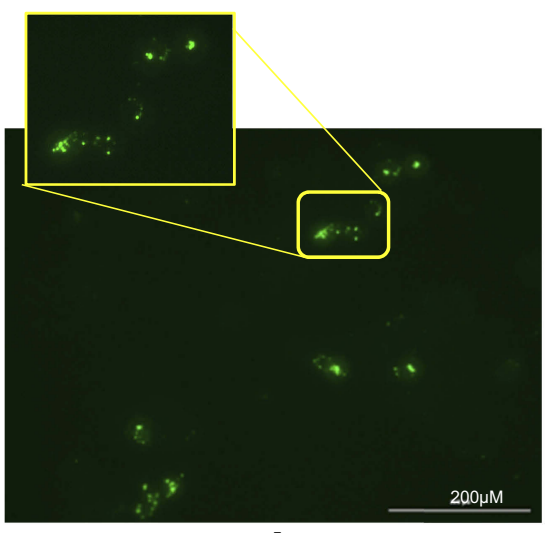

A

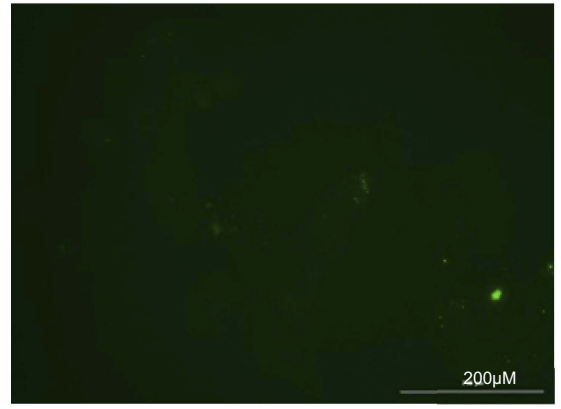

D

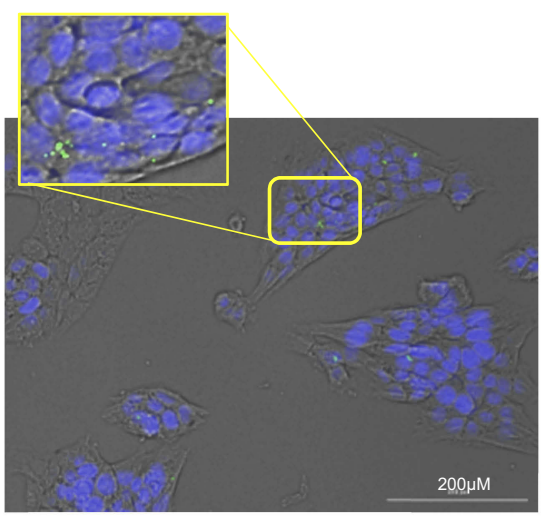

B

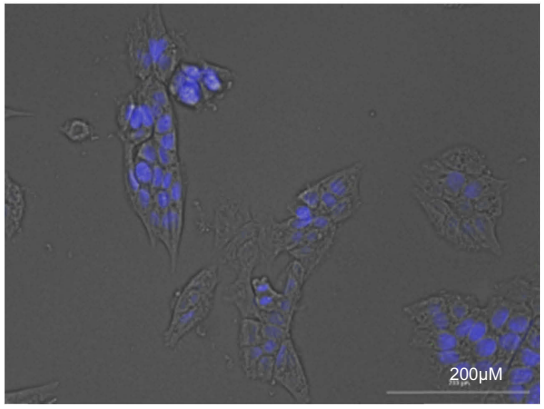

E

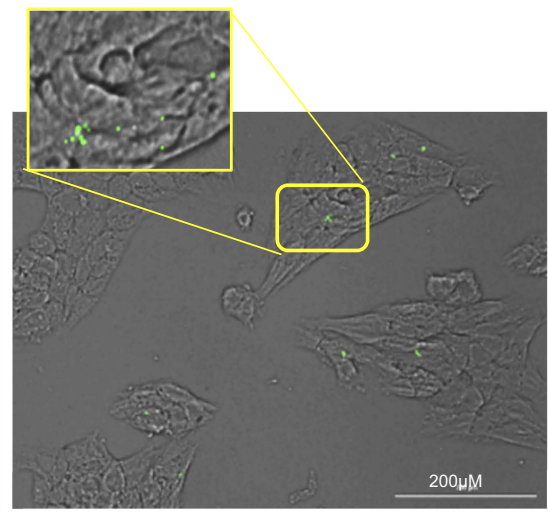

C

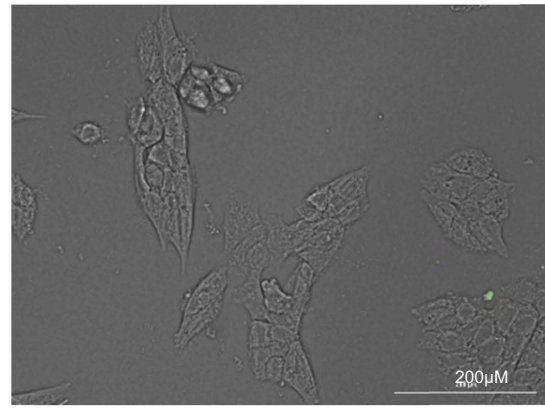

$\mathbf{F}$

Figure 4 Binding of PKH-67 labeled exosomes to TUBO cells. Exosomes isolated from MSCs were labeled with PKH-67 and incubated with TUBO cells for 24 hours. (A) Fluorescent image of binding of PKH-67 targeted exosomes which showed significantly higher binding compared to PKH-67 untargeted exosomes. (B) Merged fluorescent image of DAPI, PKH-67, and phase contrast. (C) Merged bright field and PKH-67 fluorescent images of TUBO cells. (D) Fluorescent image of untargeted exosomes. (E) Merged DAPI, PKH-67, and bright field image of untargeted exosomes. (F) Merged bright field and PKH-67 fluorescent image of untargeted exosomes binding to TUBO cells. Labeled exosomes were colored green and nuclei colored by DAPI were observed in blue.

Abbreviation: MSCs, mesenchymal stem cells.

free DOX, and PBS $(P<0.05)$ (Figure $8 \mathrm{~A})$. However, no significant difference was detected in mice weight among these groups (Figure 8B).

\section{Discussion}

Currently, the main approach of drug delivery is using artificial nanoparticles such as liposomes to encapsulate and deliver chemotherapeutic drugs to tumor sites. Despite being widely used, synthetic particles have many disadvantages, including possible induction of immune response. On the contrary, as natural vehicles for drug delivery, exosomes can evade the reticuloendothelial system and, thus, are tolerated by the immune system. Furthermore, exosomes are biocompatible and non-toxic, with the ability to entrap sufficient amounts of cargo. ${ }^{6}$ They can escape the endosomal pathway by fusing with the cell membrane. Exosomes are stable in size, structure, and drug loading before injection and during circulation in the body. However, their disadvantages include low loading efficiency and low yields from cell media, especially for industrial production. ${ }^{22}$ Clinical trials have confirmed safety and biocompatibility of exosomes in humans, introducing exosomes as good candidates for drug delivery. ${ }^{23}$

As one of the most effective chemotherapeutic drugs, ${ }^{6}$ doxorubicin causes cytotoxicity via several mechanisms, including intercalation into DNA and/or cell membrane damaging by free radicals. ${ }^{24}$ On the other hand, doxorubicin has been associated with low bioavailability and adverse side-effects such as cardiotoxicity and myelosuppression, ${ }^{6}$ as well as kidney, liver, and indirect brain toxicity. ${ }^{25}$

Toffoli et al $^{26}$ proved that exosomes loaded with doxorubicin are less toxic and can change biodistribution behavior. $^{26}$ The result of a study by Hadla et $\mathrm{al}^{23}$ corroborated with this to demonstrate less toxicity of exo-DOX compared to free DOX through reducing cardiac sideeffects of doxorubicin. Therefore, along with passive targeting resulting from EPR effect and tumor-associated leaky vasculature, ${ }^{25}$ active targeting of exosomes encapsulated with doxorubicin may prevent systemic toxicity and 

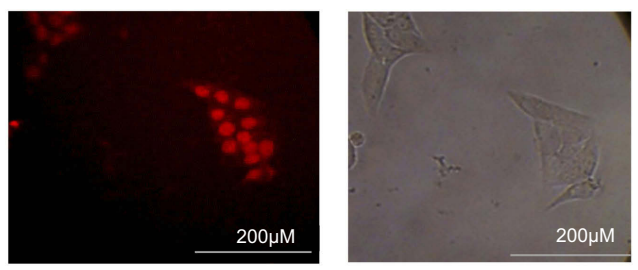

A

\section{TUBO cells}
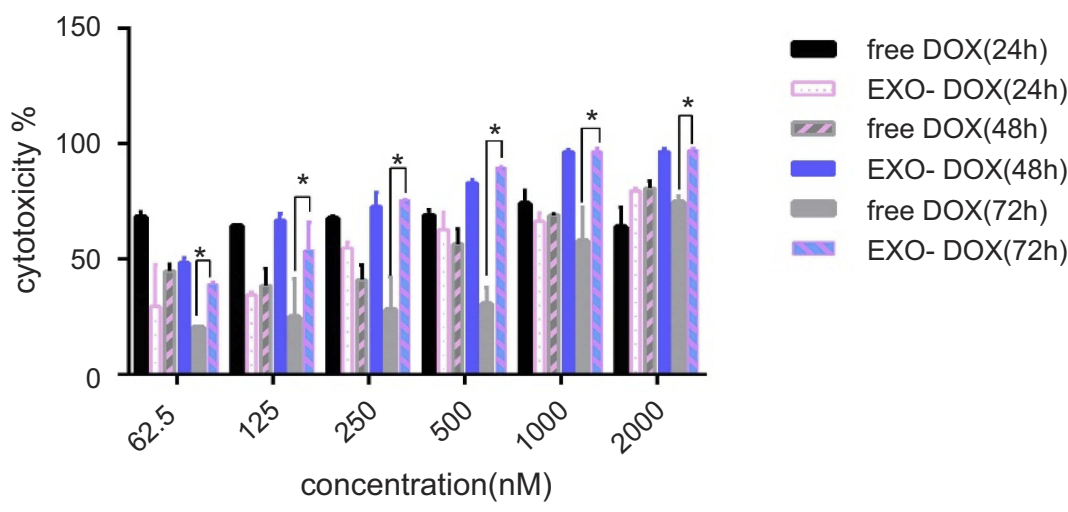

B

Figure 5 DOX delivery to TUBO cells and in vitro cellular cytotoxicity. (A) TUBO cells were incubated with $200 \mathrm{nM}$ of exo-DOX for 4 hours. (B) TUBO cells were incubated with free DOX, targeted and untargeted exo-DOX for 24 hours, 48 hours, and 72 hours. Cell cytotoxicity was assessed using MTT assay. Exo-Dox showed similar cytotoxicity with free Dox after24 hours and 48 hours, with more cytotoxicity at 72 hours. The Student's $t$-test was used to assess the significance of difference in cytotoxicity of the tested groups. Each error bar represents the mean \pm SD of three replicates. $* P<0.05$.

Abbreviations: DOX, doxorubicin; exo-DOX, doxorubicin-loaded exosome.

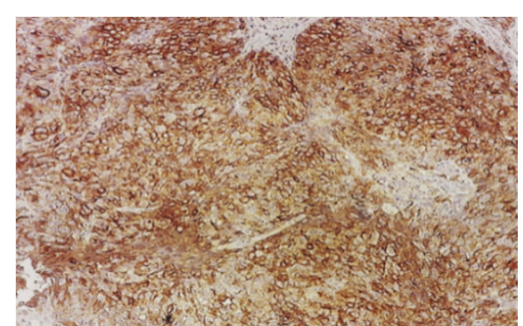

A

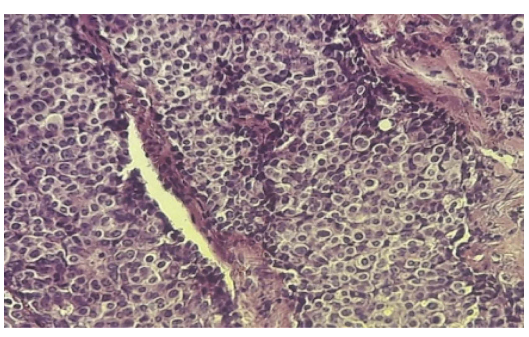

B

Figure 6 Immunohistochemistry of the TUBO tumor. (A) Brown colors indicate the HER2 receptor on the cell surface, (B) H\&E staining of TUBO tumor.

increase the efficiency of this drug, which leads to extravasation of exosomes in the tumor site. Expression of chimeric protein ligands with the affinity for specific receptors is the common method of exosome targeting, which allows for the safe use of doxorubicin in higher doses, resulting in reduced toxicity for non-target tissues and an increased potential effect of doxorubicin.

Alvarez-Erviti et al expressed RVG peptide fused to LAMP2b on the surface of dendritic cells to produce targeted exosomes and loaded them with exogenous siRNA to suppress braintumor. Tian et $\mathrm{al}^{16,22}$ engineered immature dendritic cells to express a chimeric protein containing LAMP2b fused to $\alpha v$ integrin-specific RGD peptide. Both studies indicated that expression of targeting peptides on the exosome surface increased the cellular uptake in target tissue. Ohno et $\mathrm{al}^{27}$ designed a complex chimeric protein composed of myc-tag, hemagglutinin, and GE11 or EGF on the surface of exosomes. GE11 was identified as a proper targeting peptide for tumor tissues by specifically binding EGFR without promoting the receptor.

In the present study, we used LAMP2b fused to DARPin and transduced MSCs to produce exosomes selectively targeting the HER2 antigen on breast cancer cells. In 

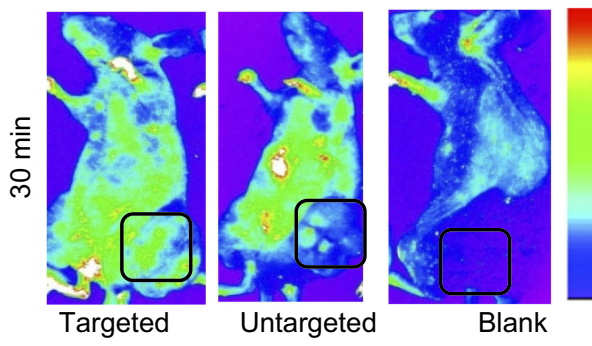

A

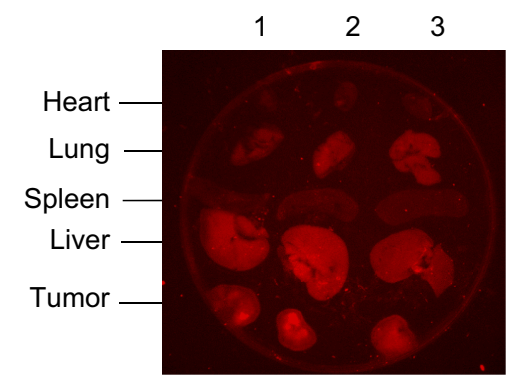

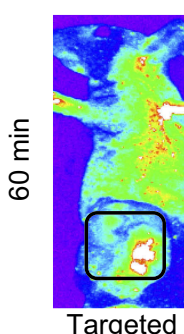

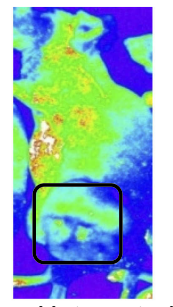

Untargeted

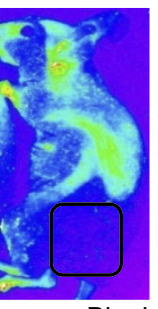

Blank

B

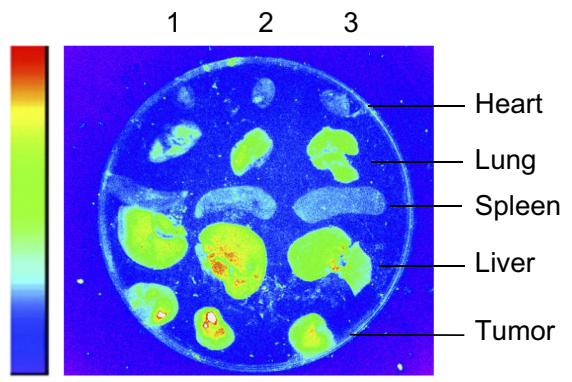

C

Figure 7 Biodistribution of exo-DOX in mice bearing TUBO tumor. In vivo targeting efficiency of targeted exo-DOX were assessed in a murine TUBO tumor model. These

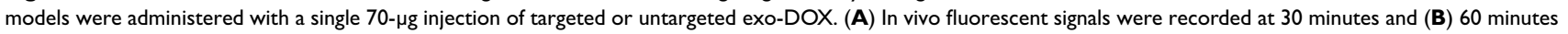
post-injection. Fluorescence was detected at the tumor sites (black boxes), with gradual increasing in signal intensity at 60 minutes. Enhanced permeability and retention (EPR) effect causes accumulation of untargeted exo-DOX in tumor site, while it was lower in comparison to targeted exo-DOX. PBS was injected as blank and no fluorescence was detected in these mice. (C) Ex vivo fluorescent imaging of major organs of the tumor model after 60 minutes. Lanes I, 2, and 3 were sequentially related to untargeted, targeted, and PBS intravenous injections. Accumulation of targeted and untargeted exo-DOX was mainly in the liver and lung, while, there was no accumulation in the heart after 60 minutes. Fluorescent intensity of untargeted exo-DOX was lower than targeted exo-DOX.

Abbreviations: DOX, doxorubicin; exo-DOX, doxorubicin-loaded exosome DOX.

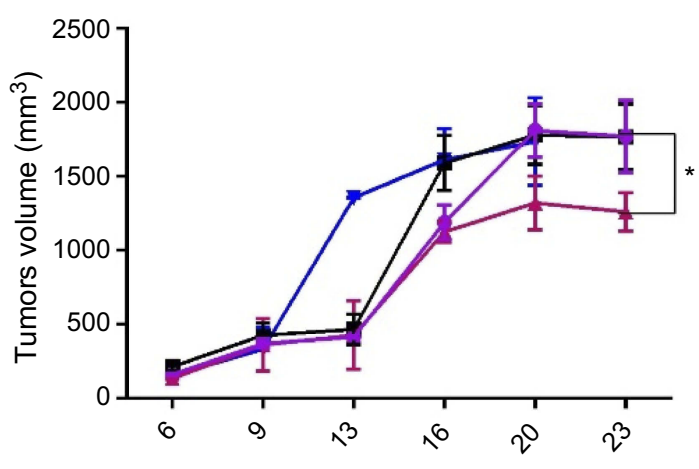

Days after tumor inoculation

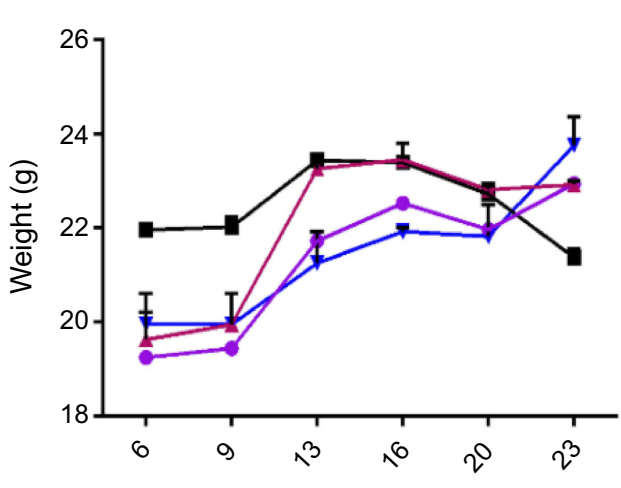

B

Figure 8 Therapeutic effects of exo-DOX in b6 nude mice bearing TUBO tumor. Mice bearing TUBO tumors $\left(>100 \mathrm{~mm}^{3}\right)$ were administrated intravenously with I.5 mg/kg of different reagents (PBS, free DOX, targeted and untargeted exo-DOX), ttwice a week for a total of six injections. (A) Tumor growth was measured by digital caliper after each injection, and the antitumor activity of each group was assessed via tumor volume measurements. Targeted exo-DOX treatment dramatically reduced the rate of tumor growth compared to the control group $\left({ }^{*} P<0.05\right)$. At the experimental dose, free DOX and untargeted exo-DOX showed no significant effects on tumor growth $(\mathbf{B})$ No significant difference was detected in body mass among the mice in these groups.

Abbreviations: DOX, doxorubicin; exo-DOX, doxorubicin-loaded exosome DOX.

a previously published study, we used this chimeric peptide for exosome targeting. ${ }^{11}$ Next, we compared the antitumor effect of the targeted and untargeted exo-DOX with free DOX in vitro on the MDA-MB231 and BT-474 cell lines. ${ }^{12}$
Herein, we further investigated the tumor inhibition effect of targeted and untargeted exo-DOX in TUBO cells in vitro and in vivo. Our results indicated a significant difference in the binding efficiency of PKH-67 labeled targeted and 
untargeted exosomes to both immobilized HER2 protein and HER2-positive SKBR3 cells. Although no significant difference was observed in binding to SKBR3 at $0.2 \mu \mathrm{g} / \mu \mathrm{L}$, which can be interpreted to be due to physical attachment and non-specific entrance of untargeted exosomes in higher concentrations. These results were confirmed by flow cytometry at increased concentrations. Furthermore, we found exo-DOX to have more anti-tumor activity compared to free DOX in vitro. This finding corroborated with the results of Yang et al, ${ }^{6}$ while contradicting the results of Tian et al ${ }^{16}$ and our previous results. ${ }^{12}$ Since the mentioned studies used 24 and 48 hours of incubation time, a possible explanation may be prolonged treatment of the cells for 72 hours, leading to enhanced stability of encapsulated exoDOX in comparison with free DOX. ${ }^{6}$

TUBO breast cancer cell line overexpressing rat HER2/neu protein was established from a lobular carcinoma which spontaneously arose in female BALB/c mice. The rHER2/neu proto-oncogene was driven by the mouse mammary tumor virus (MMTV) promoter. ${ }^{28-30}$ Although this tumor model may help to predict a reaction in the body, subcutaneous injection cannot reproduce the real tumor microenvironment, which is one of the disadvantages for these models. ${ }^{24}$

The enhanced effect of doxorubicin entrapped in targeted exosomes can be explained by a higher dosage of doxorubicin accumulated in the tumor tissue using targeted exosomes for delivery. ${ }^{24}$ We confirmed that exoDOX significantly increases the anti-cancer effect of doxorubicin against TUBO cells, by increasing the stability and/or uptake of this drug compared to the free DOX at 72 hours in vitro (Figure 5B). This was further investigated in nude mouse models, where we observed specific affinity of the exo-DOX for TUBO tumor cells and its diminishing effect on the growing rate of this tumor (Figure 8A).

Our results revealed no significant change in mice weight following treatment with $30 \mu \mathrm{g}$ per injection of free DOX $(1.5 \mathrm{mg} / \mathrm{kg})$, either in targeted or untargeted exo-DOX. This confirmed the results of Hadla et al, ${ }^{23}$ who showed that injection of 1.5 and $3 \mathrm{mg} / \mathrm{kg}$ free DOX and exo-DOX did not decrease body weight, and weight lost was observed at $>5 \mathrm{mg} / \mathrm{kg}$ DOX and exo-DOX. However, we report that tumors treated with $30 \mu \mathrm{g}$ per injection $(1.5 \mathrm{mg} / \mathrm{kg})$ of targeted exo-DOX can reduce the rate of tumor growth after 21 days of biweekly injections (Figure 8A).

Based on the literature, the healing effect of a series of IV injected DOX is succeeded at $5 \mathrm{mg} / \mathrm{kg}^{24}$ In our study, we showed that the dosage of DOX can be reduced by encapsulating in targeted exosomes and decrease the therapeutic potential dose to $1.5 \mathrm{mg} / \mathrm{kg}$. Untargeted exo-DOX and free DOX had no effect on the tumor volume, which may be due to the low dosage $(1.5 \mathrm{mg} / \mathrm{kg})$ used compared to the report published by Rudnick-Glick et al. ${ }^{24}$

\section{Conclusion}

We demonstrated that expression of chimeric LAMP2bDARPin protein on the surface of exosomes leads to more efficient binding to HER2-positive TUBO cells compared to HER2-negative 4T1cells. Moreover, this novel drug delivery system can increase accumulation of DOX in the tumor site and reduce the rate of growth in the HER2/neu-overexpressing TUBO breast cancer model. The results revealed that the targeted delivery of DOX significantly increased the effectiveness of the drug, thus lower concentrations can be administered.

\section{Abbreviations list}

DOX, doxorubicin; exo-dox, doxorubicin-loaded exosome; ECL Western blotting, enhanced chemi-luminescence; EPR, Enhanced permeability and retention; LAMP, lysosome associated membrane protein; HER2, human epidermal growth factor receptor 2; DARPins, designed ankyrin repeat proteins; DMSO, dimethyl sulfoxide; MTT, 3-(4,5-Dimethylthiazol-2Yl)-2,5-Diphenyltetrazolium Brom.

\section{Ethical approval}

All experiments in this research were done in accordance with the guidelines of the Declaration of Helsinki (1975) and The Society for Neuroscience Animal Care (1998). The guidelines used were approved for implementation by the Medical Ethics Committee, Faculty of Medical Sciences, Tarbiat Modares University, on April 17, 2006. In addition this study, including the animal experiments and use of the TUBO cell lines, was approved by Medical Ethics Committee, Faculty of Medical Sciences, Tarbiat Modares University, on April 28, 2017; approval reference number: IR.TMU.TEC.1395.5.

\section{Acknowledgments}

The authors wish to thank Tarbiat Modares University for partially funding the project. This study is a part of the $\mathrm{PhD}$ thesis of Hosna Gomari, Tarbiat Modares University, Tehran, Iran. 


\section{Disclosure}

The authors report no conflicts of interest in this work.

\section{References}

1. Hood JL. Post isolation modification of exosomes for nanomedicine applications. Nanomedicine. 2016;11(13):1745-1756. doi:10.2217/ nnm-2016-0102

2. Zhao W, Song Zhuang X-RQ. Comparative study of the in vitro and in vivo characteristics of cationic and neutral liposomes. Int $J$ Nanomedicine. 2011;6:3087. doi:10.2147/IJN.S25646

3. Zhao J, Xie P, Ye C, et al. Outside-in synthesis of mesoporous silica/ molybdenum disulfide nanoparticles for antitumor application. Chem Eng J. 2018;351:157-168. doi:10.1016/j.cej.2018.06.101

4. Yang H, Zhao J, Wu C, Ye C, Zou D, Wang S. Facile synthesis of colloidal stable MoS2 nanoparticles for combined tumor therapy. Chem Eng J. 2018;351:548-558. doi:10.1016/j.cej.2018.06.100

5. Guo B, Zhao J, Wu C, et al. One-pot synthesis of polypyrrole nanoparticles with tunable photothermal conversion and drug loading capacity. Colloids Surf B Biointerfaces. 2019;177:346-355. doi:10.1016/j.colsurfb.2019.02.016

6. Yang Y, Chen Y, Zhang F, Zhao Q, Zhong H. Increased anti-tumour activity by exosomes derived from doxorubicin-treated tumour cells via heat stress. Int $J$ Hyperthermia. 2015;31(5):498-506. doi:10.3109/02656736.2015.1036384

7. Johnsen KB, Gudbergsson JM, Skov MN, Pilgaard L, Moos T, Duroux M. A comprehensive overview of exosomes as drug delivery vehicles - endogenous nanocarriers for targeted cancer therapy. Biochim Biophys Acta Rev Cancer. 2014;1846(1):75-87. doi:10.1016/j.bbcan.2014.04.005

8. Yuan Z, Kolluri KK, Gowers KH, Janes SM. TRAIL delivery by MSC-derived extracellular vesicles is an effective anticancer therapy. $J$ Extracell Vesicles. 2017;6(1):1265291. doi:10.1080/ 20013078.2017.1265291

9. Klopp AH, Spaeth EL, Dembinski JL, et al. Tumor irradiation increases the recruitment of circulating mesenchymal stem cells into the tumor microenvironment. Cancer Res. 2007;67(24):1168711695. doi:10.1158/0008-5472.CAN-07-1406

10. Khodashenas Limoni S, Salimi F, Forouzandeh Moghaddam M. Designing pLEX-LAMP-DARPin lentiviral vector for exression of HER2 targeted DARPin on exosome surface. J Mazandaran Univ Med Sci. 2017;27(151):12-23.

11. Limoni SK, Moghadam MF, Moazzeni SM, Gomari H, Salimi F. Engineered exosomes for targeted transfer of siRNA to HER2 positive breast cancer cells. \#ABAB-D-18-00049R1, ed. Appl Biochem Biotechnol. 2019;187(1):352-364. doi:10.1007/s12010-018-2813-4

12. Gomari H, Moghadam MF, Soleimani M. Targeted cancer therapy using engineered exosome as a natural drug delivery vehicle. Onco Targets Ther. 2018;11:5753-5762. doi:10.2147/OTT

13. Jalali SA, Sankian M, Tavakkol-Afshari J, Jaafari MR. Induction of tumor-specific immunity by multi-epitope rat HER2/neu-derived peptides encapsulated in LPD nanoparticles. Nanomedicine. 2012;8 (5):692-701. doi:10.1016/j.nano.2011.09.010

14. Razazan A, Behravan J, Arab A, et al. Conjugated nanoliposome with the HER2/neu-derived peptide GP2 as an effective vaccine against breast cancer in mice xenograft model. PLoS One. 2017;12(10): e0185099. doi:10.1371/journal.pone.0185099
15. Escrevente C, Keller S, Altevogt P, Costa J. Interaction and uptake of exosomes by ovarian cancer cells. BMC Cancer. 2011;11(1):108. doi:10.1186/1471-2407-11-108

16. Tian Y, Li S, Song J, et al. A doxorubicin delivery platform using engineered natural membrane vesicle exosomes for targeted tumor therapy. Biomaterials. 2014;35(7):2383-2390. doi:10.1016/j. biomaterials.2013.11.083

17. Tan CY, Lai RC, Wong W, Dan YY, Lim S-K, Ho HK. Mesenchymal stem cell-derived exosomes promote hepatic regeneration in druginduced liver injury models. Stem Cell Res Ther. 2014;5(3):76. doi: $10.1186 /$ scrt465

18. Kim MS, Haney MJ, Zhao Y, et al. Development of exosome-encapsulated paclitaxel to overcome MDR in cancer cells. Nanomedicine. 2016;12(3):655-664. doi:10.1016/j.nano.2015.10.012

19. Li W, Mu D, Tian F, et al. Exosomes derived from Rab27a-overexpressing tumor cells elicit efficient induction of antitumor immunity. Mol Med Rep. 2013;8(6):1876-1882. doi:10.3892/ mmr.2013.1738

20. Bellavia D, Raimondo S, Calabrese G, et al. Interleukin 3-receptor targeted exosomes inhibit in vitro and in vivo chronic myelogenous leukemia cell growth. Theranostics. 2017;7(5):1333. doi:10.7150/ thno.17092

21. Rivoltini L, Chiodoni C, Squarcina P, et al. TNF-related apoptosisinducing ligand (TRAIL)-armed exosomes deliver proapoptotic signals to tumor site. Clin Cancer Res. 2016;22(14):3499-3512. doi:10.1158/1078-0432.CCR-15-2170

22. Antimisiaris S, Mourtas S, Marazioti A. Exosomes and exosomeinspired vesicles for targeted drug delivery. Pharmaceutics. 2018;10 (4):218. doi:10.3390/pharmaceutics10040218

23. Hadla M, Palazzolo S, Corona G, et al. Exosomes increase the therapeutic index of doxorubicin in breast and ovarian cancer mouse models. Nanomedicine. 2016;11(18):2431-2441. doi:10.2217/nnm-2016-0154

24. Rudnick-Glick S, Corem-Salkmon E, Grinberg I, Margel S. Targeted drug delivery of near IR fluorescent doxorubicin-conjugated poly (ethylene glycol) bisphosphonate nanoparticles for diagnosis and therapy of primary and metastatic bone cancer in a mouse model. $J$ Nanobiotechnology. 2016;14(1):80. doi:10.1186/s12951-016-0160-6

25. MacDiarmid JA, Langova V, Bailey D, et al. Targeted doxorubicin delivery to brain tumors via minicells: proof of principle using dogs with spontaneously occurring tumors as a model. PLoS One. 2016;11 (4):e0151832. doi:10.1371/journal.pone.0151832

26. Toffoli G, Hadla M, Corona G, et al. Exosomal doxorubicin reduces the cardiac toxicity of doxorubicin. Nanomedicine. 2015;10 (19):2963-2971. doi:10.2217/nnm.15.118

27. Ohno SI, Takanashi M, Sudo K, et al. Systemically injected exosomes targeted to EGFR deliver antitumor microRNA to breast cancer cells. Mol Ther. 2013;21(1):185-191. doi:10.1038/ mt.2012.180

28. Park S, Jiang Z, Mortenson ED, et al. The therapeutic effect of antiHER2/neu antibody depends on both innate and adaptive immunity. Cancer Cell. 2010;18(2):160-170. doi:10.1016/j.ccr.2010.06.014

29. Rovero S, Amici A, Di Carlo E, et al. DNA vaccination against rat her-2/Neu p185 more effectively inhibits carcinogenesis than transplantable carcinomas in transgenic BALB/c mice. $J$ Immunol. 2000;165(9):5133-5142. doi:10.4049/jimmunol.165.9.5133

30. Pierpaoli E, Viola V, Barucca A, Orlando F, Galli F, Provinciali M. Effect of annatto-tocotrienols supplementation on the development of mammary tumors in HER-2/neu transgenic mice. Carcinogenesis. 2013;34(6):1352-1360. doi:10.1093/carcin/bgt064 


\section{Publish your work in this journal}

The International Journal of Nanomedicine is an international, peerreviewed journal focusing on the application of nanotechnology in diagnostics, therapeutics, and drug delivery systems throughout the biomedical field. This journal is indexed on PubMed Central, MedLine, CAS, SciSearch ${ }^{\circledR}$, Current Contents ${ }^{\circledR} /$ Clinical Medicine,
Journal Citation Reports/Science Edition, EMBase, Scopus and the Elsevier Bibliographic databases. The manuscript management system is completely online and includes a very quick and fair peer-review system, which is all easy to use. Visit http://www.dovepress.com/ testimonials.php to read real quotes from published authors. 